This item was submitted to Loughborough's Research Repository by the author.

Items in Figshare are protected by copyright, with all rights reserved, unless otherwise indicated.

\title{
Semantic transfer and contradictory evidence in intuitionistic fuzzy sets
}

PLEASE CITE THE PUBLISHED VERSION

PUBLISHER

(C) IEEE

VERSION

VoR (Version of Record)

LICENCE

CC BY-NC-ND 4.0

\section{REPOSITORY RECORD}

Hinde, Chris J., Robert S. Patching, and Stephen A. McCoy. 2019. "Semantic Transfer and Contradictory Evidence in Intuitionistic Fuzzy Sets". figshare. https://hdl.handle.net/2134/4120. 
This item was submitted to Loughborough's Institutional Repository (https://dspace.lboro.ac.uk/) by the author and is made available under the following Creative Commons Licence conditions.

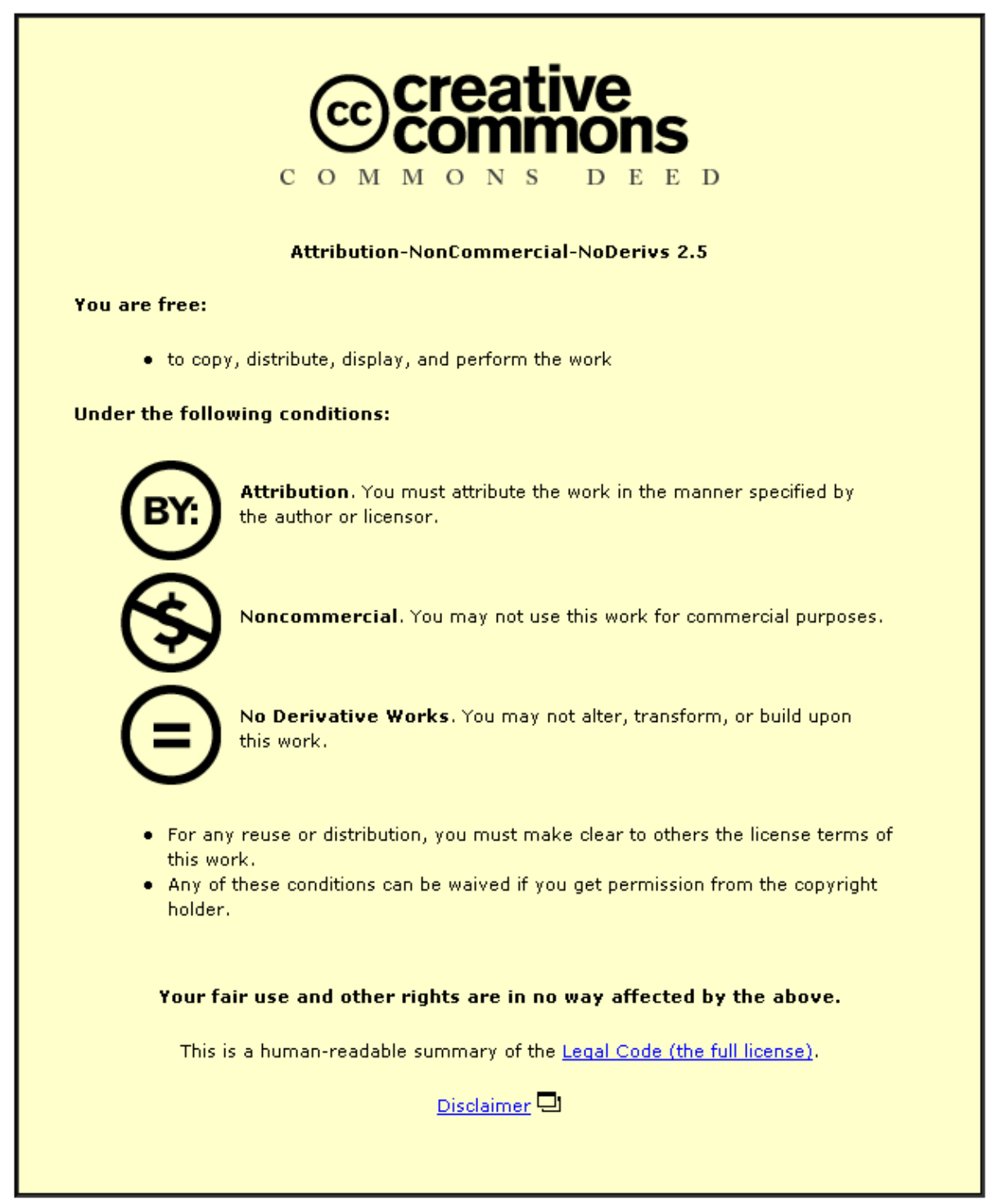

For the full text of this licence, please go to: http://creativecommons.org/licenses/by-nc-nd/2.5/ 


\title{
Semantic Transfer and Contradictory Evidence in Intuitionistic Fuzzy Sets
}

\author{
C.J. Hinde, R.S. Patching and S.A. McCoy
}

\begin{abstract}
The relationship between object level intuitionistic fuzzy sets and predicate based intuitionistic fuzzy sets is explored. Mass assignment uses a process called semantic unification to evaluate the degree to which one set supports another, the inverse function is semantic separation. Intuitionistic fuzzy sets are mapped onto a mass assignment framework and the semantic unification operator is generalised to support both mass assignment and intuitionistic fuzzy sets, as is semantic separation. Transfer of inconsistent and contradictory evidence are also dealt with.
\end{abstract}

Keywords: Inconsistency, Contradiction, Intuitionistic Fuzzy Sets, Mass Assignment, Semantic Unification, Conditionalisation, Semantic Separation, Abduction.

\section{Introduction}

$\mathbf{T}$ HE correspondence between the representation of fuzziness in intuitionistic fuzzy sets, Atanassov $(1 ; 2 ; 3)$ and that used in mass assignment (4) is used to explore the generalisation of semantic unification and separation used in mass assignment and introduce them to intuitionistic fuzzy sets. It begin by using the semantic unification and separation operators developed by Patching (5) and Hinde (6). This research concentrated on the inconsistency that arises from evidence that removes all possibilities and how to deal with that inconsistency. Later work by Hinde (7) explored the effect of incorporating a measure of contradiction into the framework of intuitionistic fuzzy sets. Subsequently a more thorough analysis (8) related intuitionistic fuzzy sets to mass assignment. Subsequently in Hinde (9) this was extended and refined, defining the operations of both $\cap$ and $\wedge$, which indirectly defined $\cup$ and $\vee$. This paper provided a more thorough treatment of contradiction than was given in (8).

\section{A. Overview}

Intuitionistic fuzzy set theory is briefly introduced although the qualitative nature of the evidence resulting in the membership and non-membership values is omitted as it may be found in other papers $(8 ; 9)$. Mass assignment is extended to map Intuitionistic Fuzzy Sets and Mass assignment (8). The background to Semantic Unification is briefly introduced and then using the parallels in $(8 ; 9)$ Semantic Unification

C.J. Hinde is with the Department of Computer Science, Loughborough University, Loughborough, LE11 3TU, UK (phone: +44 1509635702 ; email: c.j.hinde@lboro.ac.uk).

R.S. Patching is with the Department of Computer Science, Loughborough University, Loughborough, LE11 3TU, UK (email: research@woollythinking.co.uk).

S.A. McCoy is with Department of Computer Science, Loughborough University, Loughborough, LE11 3TU, UK (email: S.A.McCoy@lboro.ac.uk). is explored introducing a new method of calculating the non-membership values. Semantic Separation is the inverse function is given a similar treatment. The versions used are drawn from $(5 ; 6)$ which extend the operations defined in (4).

\section{B. Intuitionistic fuzzy sets}

Intuitionistic fuzzy set theory was introduced by the seminal work of Atanassov (1), and are a generalisation of the classic fuzzy sets using a measure of membership and a measure of non-membership. The definition of an intuitionistic L-fuzzy set (ILFS) $A^{*}$ over a universe of discourse $\mathbb{U}$ is:

$$
A^{*}=\left\{\left\langle x, \mu_{A}(x), \nu_{A}(x)\right\rangle \mid x \in \mathbb{U}\right\}
$$

subject to

$$
\mu_{A}(x)+\nu_{A}(x) \leq 1 \mid x \in \mathbb{U}
$$

Following the notation of Atanassov, Hinde (7) defined inconsistent intuitionistic fuzzy sets, IIFS.

$$
A^{\iota *}=\left\{\left\langle x, \mu_{A}(x), \nu_{A}(x), \iota_{A}(x)\right\rangle \mid x \in \mathbb{U}\right\}
$$

subject to

$$
\mu_{A}(x)+\nu_{A}(x)+\iota_{A}(x) \leq 1 \mid x \in \mathbb{U}
$$

Inconsistent Intuitionistic Fuzzy Sets are able to represent a lack of knowledge that arises from a variety of circumstances. Hinde (7) explored some of these.

Subsequently (8) this was mapped across to a mass assignment representation where an element of mass, defined in (4) as a scalar, was extended to be a triple. The triple vector of mass allowed the representation of a membership value, a non-membership value and a value for the contradiction (8) brought about by evidence, from the same source, for membership and non-membership.

\section{Relationship to Mass assignment}

An exploration of the relationship to mass assignment is given in (8). The main contribution which shall be used here is the representation of mass as a triple:

$\mathrm{m}_{A}(X)=\left\langle\mu_{A}(X), \nu_{A}(X), \iota_{A}(X)\right\rangle$

This allows several possible denotations of contradiction, as enumerated below:

1) $\mathrm{m}_{A}(\{\})=(\delta, 0,0)$

- contradiction arising from the membership curve, where all possibilities have been eliminated.

2) $\mathrm{m}_{A}(\mathbb{U})=(0, \delta, 0)$

- contradiction arising from the non-membership curve, stating that all members of the support set have evidence for non-membership. 
3) $\mathrm{m}_{A}(X)=(0,0, \delta)$

- contradiction arising from contradictory evidence about the subset $\mathrm{X}$ where the evidence is for membership and also non-membership.

Selection functions are needed to extract sub masses so the equations can be sensibly expressed, see Equation 4 .

$$
\begin{aligned}
\mathrm{m}_{A}(X)= & \left\langle\mu_{A}(X), \nu_{A}(X), \iota_{A}(X)\right\rangle \\
\mu\left(\mathrm{m}_{A}(X)\right)=\mu_{A}(X) & \nu\left(\mathrm{m}_{A}(X)\right)=\nu_{A}(X) \\
\iota\left(\mathrm{m}_{A}(X)\right)= & \iota_{A}(X)
\end{aligned}
$$

The notation here has used the quantities $\mu, \nu$ and $\iota$ as projection operators to extract the values $\mu, \nu$ and $\iota$ from the mass triples. Although this is overloading we believe this is better as it is clear what they are extracting.

\section{Votes for and against}

Fuzzy sets can be built up using a voting model, (10) giving a membership curve, and similarly a non-membership curve, and potentially contradictory votes. These are counted in the triple mass assignment. Once all votes have been cast we should be in a position to calculate the contradiction, and also the ignorance. However, without knowing whether a vote has been cast twice or not at all it would not be possible to determine which was cast. Figure 1 shows the membership and non-membership curve for the assignment shown in Equation 5.

$$
\begin{aligned}
\mathrm{m}_{A}(\{\})=\langle 0.1,0.6,0.0\rangle & \mathrm{m}_{A}(\{a\})=\langle 0.2,0.0,0.1\rangle \\
\mathrm{m}_{A}(\{a, b\})=\langle 0.1,0.0,0.0\rangle & \mathrm{m}_{A}(\{c\})=\langle 0.0,0.1,0.0\rangle \\
\mathrm{m}_{A}(\{b, c\})=\langle 0.0,0.2,0.0\rangle & \operatorname{m}_{A}(\{a, b, c\})=\langle 0.5,0.0,0.0\rangle
\end{aligned}
$$

There are votes for the sets $\{a\},\{a, b\}$ and $\{a, b, c\}$, which give rise to Equation 6, the membership curve mass assignment. The votes against are represented in Equation 7, the non-membership curve mass assignment. Contradictory votes are in Equation 8.

$$
\begin{array}{cl}
\mathrm{m}_{A}(\{\})=0.1 & \mathrm{~m}_{A}(\{a\})=0.2 \\
\mathrm{~m}_{A}(\{a, b\})=0.1 & \mathrm{~m}_{A}(\{a, b, c\})=0.5 \\
\operatorname{m}_{A}(\{\})=0.6 & \mathrm{~m}_{A}(\{c\})=0.1 \\
\operatorname{m}_{A}(\{b, c\})=0.2 & \\
\mathrm{~m}_{A}(\{a\})=0.1
\end{array}
$$

The mass assignment of the possibility curve derived from the non-membership curve is derived from taking the complement of the mass supports, Equation 9, giving Equation 10. But correctly votes assigned to contradiction

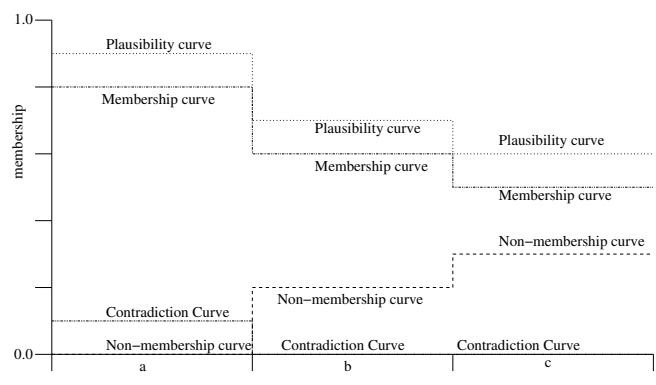

Fig. 1. Showing the four curves, the membership curve, the nonmembership curve, the corresponding possibility curve arising from it and the contradiction curve; all arising from the mass assignment in Equation 5.

cannot be assigned to membership so the possibility curve would be as shown in Equation 11

$$
\begin{aligned}
\mathrm{m}_{A}(\bar{X})=\nu\left(\mathrm{m}_{A}(X)\right) & \\
\mathrm{m}_{A}(\{a, b, c\}) & =0.6 \\
\mathrm{~m}_{A}(\{a, b\}) & =0.1 \quad \mathrm{~m}_{A}(\{a\})=0.2 \\
\mathrm{~m}_{A}(\{a, b, c\}) & =0.6 \\
\mathrm{~m}_{A}(\{a, b\}) & =0.1 \quad \mathrm{~m}_{A}(\{a\})=0.2
\end{aligned}
$$

It is now relatively straightforward to calculate two types of ignorance. There is the ignorance that exists as a result of no votes being cast, where the mass is all held in the set of support for both the membership curve and the possibility curve derived from the non-membership curve. There is the value of $\pi$ that is the difference between the membership curve and the complement of the non-membership curve. Calculating values of $\mu, \nu$ and $\pi$ for the 3 elements results in:

$$
\begin{aligned}
& \mu(a)=1.0, \neg \nu(a)=1.0, \pi(a)=0.0 \\
& \mu(b)=0.8, \neg \nu(b)=0.9, \pi(b)=0.1 \\
& \mu(c)=0.6, \neg \nu(c)=0.7, \pi(c)=0.1
\end{aligned}
$$

These values are constrained by Equations 12 .

$$
\begin{aligned}
& \sum \mu(\mathrm{m}(X))+\frac{\sum \iota(\mathrm{m}(X))}{2}=1 \\
& \sum \nu(\mathrm{m}(X))+\frac{\sum \iota(\mathrm{m}(X))}{2}=1
\end{aligned}
$$

IV. Inconsistent and contradictory evidence.

One sort of inconsistency has already been dealt with, that which arises from assignment of mass to the empty set \{\}. Typically the non-membership curve, when expressed as a mass assignment, has mass assigned to the empty set if the possibility curve is to have mass assigned to ignorance. 
So the non-membership curve starts off with all the mass assigned to \{\} and moves it upwards. As this is complemented it serves to produce a possibility curve. Inconsistency is mass assigned to $\mathbb{U}$ in the non-membership curve and corresponds to mass assigned to \{\} in the membership curve. Contradiction is mass assigned to both the membership curve and the non-membership curve. Cubillo (11) measures self contradiction in Intuitionistic Fuzzy Sets but does not consider that contradiction may arise before the set is complete. So the applicable constraint is now as shown in Equation 13. In Cubillo's terms a nonzero value of $\iota_{A}(x)$, contradiction, would imply a zero value of $\pi_{A}(x)$, hesitation; here we allow all four values to be non-zero.

$$
\mu_{A}(x)+\nu_{A}(x)+\iota_{A}(x)+\pi_{A}(x)=1 \mid x \in \mathbb{U}
$$

\section{A. Contradiction examples.}

We now illustrate some mass assignment examples following our definition of contradiction given above and also in (7) with the corresponding belief and possibility curves that arise.

1) Inconsistent due to contradiction between types of evidence.: The masses here have a contradiction between the membership and the non-membership curve arising from votes for membership and votes for non membership from the same event; the example illustrated is taken from Equation 5. The mass assignment for the membership curve is given in Equation 14, which sums to 0.9 .

$$
\begin{aligned}
& \mathrm{m}_{A}(\{\})=0.1 \quad \mathrm{~m}_{A}(\{a\})=0.2 \\
& \mathrm{~m}_{A}(\{a, b\})=0.1 \quad \mathrm{~m}_{A}(\{a, b, c\})=0.5
\end{aligned}
$$

It is tempting to make the sum of the mass elements total 1.0 by either adding a mass of 0.1 to \{\} or $\{a, b, c\}$; but that does not represent where the votes have gone correctly. The non-membership mass assignment in this case could be as given in Equation 15.

$$
\begin{aligned}
\mathrm{m}_{A}(\{\}) & =0.6 \\
\mathrm{~m}_{A}(\{a\}) & =0.1 \quad \mathrm{~m}_{A}(\{a, b\})=0.2
\end{aligned}
$$

Let the balance be due to contradictory votes for membership and non-membership concerning the set $\{a\}$. So there is contradictory evidence about the membership and non-membership curve for $\{a\}$. Furthermore there is ignorance about the remaining votes for membership and nonmembership curves as there is mass assigned to the set $\{a, b, c\}$ or ( $\mathrm{U})$ corresponding to uncast votes. There is inconsistency in that some votes have eliminated all options and so there is also mass assigned to \{\} . There are also votes cast for the non-membership curve that all possible elements have had positive evidence for their falsity. All votes are accounted for, some have not been cast, some are inconsistent and some have been cast twice.
Equation 5 has mass assigned to contradiction associated with the set $\{a\}$, but if contradictory votes have been cast then perhaps there should be mass assigned to the contradictory part of the complementary set $\{b, c\}$ ? However, what this states is that there have been contradictory votes cast about the set $\{a\}$.

Equation 5 has most aspects that we are interested in, the relevant curves are shown in Figure 1. We can extract all the relevant data from this to produce the normal, but inconsistent, fuzzy set:

$\{0.9|a+0.6| b+0.5 \mid c\}$

The intuitionistic fuzzy memberships:

$\{\langle 0.9,0.0\rangle|a+\langle 0.6,0.2\rangle| b+\langle 0.5,0.3\rangle \mid c\}$

The inconsistent intuitionistic fuzzy memberships:

$\{\langle 0.9,0.0,0.1\rangle|a+\langle 0.6,0.2,0.0\rangle| b+\langle 0.5,0.3,0.0\rangle \mid c\}$

This gives a unifying semantics to mass assignment and intuitionistic fuzzy sets, and introduces the ability to manage contradictory evidence.

That the mass assignment triple is a true generalisation is easily shown by the following mass assignment triple in Equation 16. All the curves in Figure 1, and the corresponding assignments above are unchanged but the entries for \{\} , $\{b\}$ and $\{c\}$ have changed.

$$
\begin{aligned}
\mathrm{m}_{A}(\{\}) & =\langle 0.1,0.4,0.0\rangle & & \mathrm{m}_{A}(\{a\})=\langle 0.2,0.0,0.1\rangle(16) \\
\mathrm{m}_{A}(\{a, b\}) & =\langle 0.1,0.0,0.0\rangle & & \mathrm{m}_{A}(\{c\})=\langle 0.0,0.3,0.0\rangle \\
\mathrm{m}_{A}(\{b\}) & =\langle 0.0,0.2,0.0\rangle & & \mathrm{m}_{A}(\{a, b, c\})=\langle 0.5,0.0,0.0\rangle
\end{aligned}
$$

B. Mass assignment operations.

The operations on the new mass measure follow the operations defined in (7), the tabular form as defined in (4) is applied to the quantities $\mu_{A}(x)$ and $\mu_{A}(x)+\iota_{A}(x)$ together with the corresponding quantities derived from $\nu_{A}(x)$. So the contradiction is propagated through the reasoning system accordingly. The mass triples cannot be derived in a single table but membership calculations require a table to perform the operations without contradiction, and another table to perform the operations including contradiction; similarly for non-membership. After collecting the resultant masses together the contradiction is calculated by subtracting the mass results without contradiction from those with contradiction and taking the maximum contradiction values for each mass block. This follows the operations defined in (7), which describes the necessary technology to perform the usual set theoretic operations. This paper moves the formalism forward.

\section{Semantic Unification.}

Semantic unification requires the definition of the semantic unification values to obtain the membership values, but also requires a commensurate operation to be defined to obtain the non-membership values. Given that the mass assignment triple has projection operators we need a further set of projection operators to extract complete single valued mass assignments over the universe of discourse from complete 
mass assignment sets over the triples. Again we overload the operators.

$$
\begin{aligned}
\mathrm{M}(A)= & \left\{\left\langle\mu_{A}(X), \nu_{A}(X), \iota_{A}(X)\right\rangle \mid X \in A,(17)\right. \\
& \left.\mu_{A}(X)>0, \nu_{A}(X)>0, \iota_{A}(X)>0\right\} \\
\mu(\mathrm{M}(A))= & \left\{\mu_{A}(X) \mid X \in A, \mu_{A}(X)>0\right\} \\
\iota(\mathrm{M}(A))= & \left\{\iota_{A}(X) \mid X \in A, \iota_{A}(X)>0\right\} \\
\nu \iota(\mathrm{M}(A))= & \left\{\nu_{A}(X)+\iota_{A}(X) \mid X \in A,\right. \\
& \left.\nu_{A}(X)+\iota_{A}(X)>0\right\}
\end{aligned}
$$

In order to correctly project the masses of the two sets $A$ and $B$ we need to form 4 mass assignment operations. The Unification operation on the memberships is the usual mass assignment operation performed on the respective sets of single membership mass assignments extracted from the sets of triple mass assignments, before analysing the supplementary operation we continue with the necessary definitions. Let the function SUP construct the supremum of two sets of mass assignments, define the operations:

| denote the operation of semantic unification

$\left.\right|_{\mu} \quad$ denote the operation of semantic unification delivering the memberships

$\left.\right|_{\mu \iota} \quad$ denote the operation of semantic unification delivering the memberships including contradiction

$\left.\right|_{\neg \nu \iota} \quad$ denote the operation of semantic unification delivering the non-memberships including contradiction based on the complements of the non-memberships

and others similarly

we then get:

$$
\begin{aligned}
\mu(\mathrm{M}(A \mid B))= & \left.\mu(\mathrm{M}(A))\right|_{\mu} \mu(\mathrm{M}(B)) \\
\nu \iota(\mathrm{M}(A \mid B))= & \left.\nu \iota(\mathrm{M}(A))\right|_{\nu} \nu \iota(\mathrm{M}(B)) \\
\nu \iota^{\prime}(\mathrm{M}(A \mid B))= & \nu \iota\left(\operatorname{m}_{\left.A\right|_{\nu} B}(X)\right)-\nu\left(\operatorname{m}_{\left.A\right|_{\nu} B}(X)\right) \\
& |X \in A| B\} \\
\iota(\mathrm{M}(A \mid B))= & S U P\left(\mu \iota^{\prime}(\mathrm{M}(A \mid B)), \nu \iota^{\prime}(\mathrm{M}(A \mid B))\right)
\end{aligned}
$$

Other quantities are defined similarly.

We can then form the mass assignment triples of the combination from these assignments. The set defined by $\mu(\mathrm{M}(A \mid B))$ contains the membership values, $\nu(\mathrm{M}(A \mid$ $B))$ the non-membership values and $\iota(\mathrm{M}(A \mid B))$ the contradictory evidence. Note that the contradictory evidence cannot be calculated from the sets, but is obtained from the individual mass assignments projected down. The operation $\left.\right|_{\nu}$ is the one that required discussion. The operation $\left.\right|_{\mu}$ is identical to the one that would be used in mass assignment and could be any one of the operators defined in $(4 ; 5 ; 6)$, depending upon application and requirements. $(5 ; 6)$ both deal with inconsistency, however (5) transfers the inconsistency into the reasoning system. (6) transfers a statement about inconsistency into the reasoning system. This paper's approach transfers contradiction into the reasoning system. The operator used in (5) is shown in Figure 2.

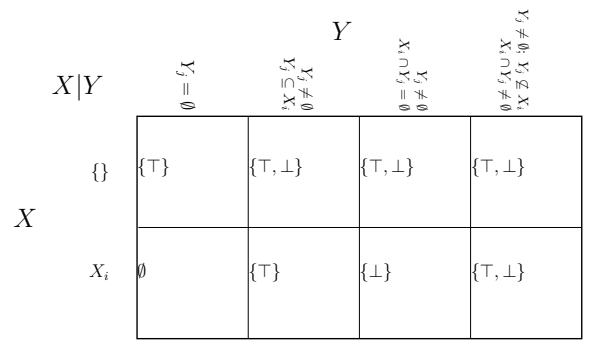

Fig. 2. Showing the tableau for the semantic unification operator.

1) Transfer of the non-membership values: Intersection is a t-norm with union the corresponding s-norm and so we can use union (1) to combine the non-membership values while performing an intersection, and vice versa. Semantic unification does not have a complementary operation and so we have to consider how the operation can be used to perform correctly on the non-membership values. The non-memberships are based on necessary evidence so the complement of this will be possibilities. This possibility curve is amenable to semantic unification, if the result of this is then complemented the result will be the new nonmemberships. If the semantic unification operator used on unit mass assignments is defined as | then Equation 19 defines some of the necessary relationships.

$$
\begin{array}{rll}
\left.\mu(\mathrm{M}(A))\right|_{\mu} \mu(\mathrm{M}(B)) & \triangle & \mu(\mathrm{M}(A)) \mid \mu(\mathrm{M}(B)) \\
\left.\mu \iota(\mathrm{M}(A))\right|_{\mu \iota} \mu \iota(\mathrm{M}(B)) & \triangle & \mu \iota(\mathrm{M}(A)) \mid \mu \iota(\mathrm{M}(B)) \\
\left.\nu(\mathrm{M}(A))\right|_{\nu} \nu(\mathrm{M}(B)) & \triangle & \neg(\neg \nu(\mathrm{M}(A) \mid \neg \nu(\mathrm{M}(B))) \\
\left.\nu \iota(\mathrm{M}(A))\right|_{\nu \iota} \nu \iota(\mathrm{M}(B)) & \triangle & \neg(\neg \nu \iota(\mathrm{M}(A)) \mid \neg \nu \iota(\mathrm{M}(B)))
\end{array}
$$

\section{Semantic Separation.}

Semantic separation, or abduction, requires the definition of the semantic separation values to obtain the membership values, but also requires a commensurate operation to be defined to obtain the non-membership values, as in Semantic unification.

In order to correctly project the masses of the two sets $A$ and $B$ we need to form 4 mass assignment operations. The Unification operation on the memberships is the usual mass assignment operation performed on the respective sets of single membership mass assignments extracted from the sets of triple mass assignments, before analysing the supplementary operation we continue with the necessary definitions. Let the function SUP construct the supremum of two sets of mass assignments, let the operations:

$\uparrow \quad$ denote the operation of semantic separation

$\uparrow_{\mu} \quad$ denote the operation of semantic separation delivering the memberships

$\uparrow_{\mu \iota} \quad$ denote the operation of semantic separation delivering the memberships including contradiction

$\uparrow_{\neg \nu} \quad$ denote the operation of semantic separation delivering the possibilities based on the complements of the non-memberships 
and others similarly

we then get:

$$
\begin{aligned}
\mu(\mathrm{M}(A \uparrow B))= & \mu(\mathrm{M}(A)) \uparrow_{\mu} \mu(\mathrm{M}(B)) \\
\mu \iota(\mathrm{M}(A \uparrow B))= & \mu \iota(\mathrm{M}(A)) \uparrow_{\mu} \mu \iota(\mathrm{M}(B)) \\
\mu \iota^{\prime}(\mathrm{M}(A \uparrow B))= & \left\{\mu \iota\left(\operatorname{m}_{A \uparrow_{\mu} B}(X)\right)-\mu\left(\operatorname{m}_{A \uparrow_{\mu} B}(X)\right)\right. \\
& \uparrow X \in A \uparrow B\} \\
& \uparrow X \in A \uparrow B\} \\
\iota(\mathrm{M}(A \uparrow B))= & S U P\left(\mu \iota^{\prime}(\mathrm{M}(A \uparrow B)), \nu \iota^{\prime}(\mathrm{M}(A \uparrow B))\right)
\end{aligned}
$$

and others similarly.

The mass assignment triples of the combination are formed from these assignments. The operation $\uparrow_{\mu}$ is identical to the one that would be used in mass assignment and could be any one of the operators defined in $(4 ; 5 ; 6)$, depending upon application and requirements. $(5 ; 6)$ both deal with inconsistency, however (5) transfers the inconsistency into the reasoning system. Hinde (6) transfers a statement about inconsistency into the reasoning system, this paper uses the simpler definition of separation, Figure 3.

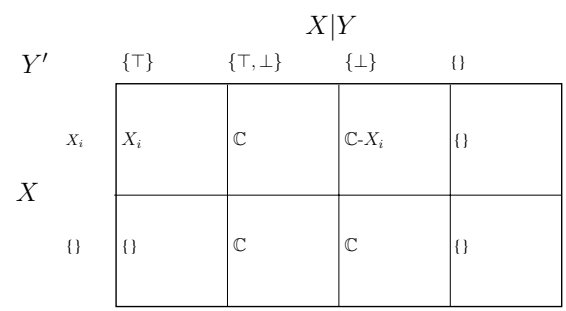

Fig. 3. Showing the tableau for the semantic separation operator.

1) Transfer of the non-membership values: Semantic separation does not have a complementary operation either and so we have the same problem and can employ the same solution. Define the semantic separation operator used on unit mass assignments as $\uparrow$, and others similarly.

$$
\begin{aligned}
& \mu(\mathrm{M}(A)) \uparrow_{\mu} \mu(\mathrm{M}(B)) \triangle \mu(\mathrm{M}(A)) \uparrow \mu(\mathrm{M}(B)) \\
& \mu \iota(\mathrm{M}(A)) \uparrow_{\mu \iota} \mu \iota(\mathrm{M}(B)) \triangleq \mu \iota(\mathrm{M}(A)) \uparrow \mu \iota(\mathrm{M}(B)) \\
& \nu(\mathrm{M}(A)) \uparrow_{\nu} \nu(\mathrm{M}(B)) \quad \triangle \neg(\neg \nu(\mathrm{M}(A) \uparrow \neg \nu(\mathrm{M}(B))) \\
& \nu \iota(\mathrm{M}(A)) \uparrow_{\nu \iota} \nu \iota(\mathrm{M}(B)) \triangleq \neg(\neg \nu \iota(\mathrm{M}(A)) \uparrow \neg \nu \iota(\mathrm{M}(B))) \\
& \text { V. Examples }
\end{aligned}
$$

The examples section will illustrate the operations of semantic unification and separation.

\section{A. Semantic Unification}

Starting with the mass assignment in Equation 5 we evaluate how well this unifies or supports the mass assignment in Equation 21.

$$
\begin{aligned}
\mathrm{m}_{B}(\{\}) & =\langle 0.1,0.6,0.0\rangle & & \mathrm{m}_{B}(\{a\})=\langle 0.2,0.0,0.1\rangle(21) \\
\mathrm{m}_{B}(\{a, b\}) & =\langle 0.1,0.0,0.0\rangle & & \mathrm{m}_{B}(\{c\})=\langle 0.1,0.1,0.0\rangle \\
\mathrm{m}_{B}(\{b, c\}) & =\langle 0.0,0.2,0.0\rangle & & \operatorname{m}_{B}(\{a, b, c\})=\langle 0.4,0.0,0.0\rangle
\end{aligned}
$$

The membership values for Equations 5 and 21

$$
\begin{aligned}
\text { Equation 5 } & \text { Equation 21 } \\
\mu \mathrm{m}_{A}(\{\})=0.1 & \mu \mathrm{m}_{B}(\{\})=0.1 \\
\mu \mathrm{m}_{A}(\{a\})=0.2 & \mu \mathrm{m}_{B}(\{a\})=0.2 \\
\mu \mathrm{m}_{A}(\{a, b\})=0.1 & \mu \mathrm{m}_{B}(\{a, b\})=0.1 \\
\mu \mathrm{m}_{A}(\{a, b, c\})=0.5 & \mu \mathrm{m}_{B}(\{a, b, c\})=0.4
\end{aligned}
$$

The unification of the membership mass assignments is shown in Figure 4.

$B$

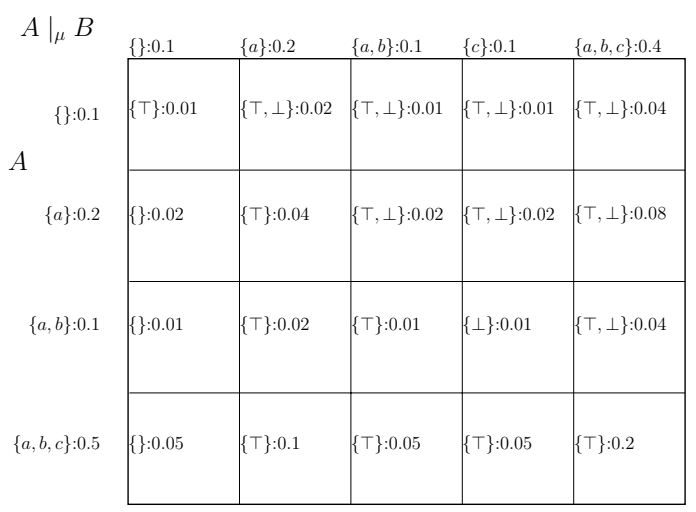

Fig. 4. Showing the tableau for the semantic unification of the membership values of the two mass assignments in Equations 5 and 21.

The mass assignment resulting from the separation of the membership functions is:

$$
\begin{aligned}
\mu \mathrm{m}_{A \uparrow B}(\{\}) & =0.08 & & \mu \operatorname{m}_{A \uparrow B}(\{T\})=0.48 \\
\mu \operatorname{m}_{A \uparrow B}(\{F\}) & =0.01 & & \mu \operatorname{m}_{A \uparrow B}(\{T, F\})=0.24
\end{aligned}
$$

The membership functions including contradictory evidence for Equations 5 and 21:

$$
\begin{aligned}
\text { Equation 5 } & \text { Equation 21 } \\
\mu \iota \mathrm{m}_{A}(\{\})=0.1 & \mu \iota \mathrm{m}_{B}(\{\})=0.1 \\
\mu \iota \mathrm{m}_{A}(\{a\})=0.3 & \mu \iota \mathrm{m}_{B}(\{a\})=0.3 \\
\mu \iota \mathrm{m}_{A}(\{a, b\})=0.1 & \mu \iota \mathrm{m}_{B}(\{a, b\})=0.1 \\
& \mu \iota \mathrm{m}_{B}(\{c\})=0.1 \\
\mu \iota \mathrm{m}_{A}(\{a, b, c\})=0.5 & \mu \iota \mathrm{m}_{B}(\{a, b, c\})=0.4
\end{aligned}
$$


$B$

\begin{tabular}{|c|c|c|c|c|c|}
\hline$\left.A\right|_{\mu \iota} B$ & \{\}$: 0.1$ & $\{a\}: 0.3$ & $\{a, b\}: 0.1$ & $\{c\}: 0.1$ & $\{a, b, c\}: 0.4$ \\
\hline\{\}$: 0.1$ & $\{T\}: 0.01$ & $\{\top, \perp\}: 0.03$ & $\{\top, \perp\}: 0.01$ & $\{T, \perp\}: 0.01$ & $\{\mathrm{~T}, \perp\}: 0.04$ \\
\hline \multicolumn{6}{|l|}{$A$} \\
\hline$\{a\}: 0.3$ & \{\}$: 0.03$ & $\{T\}: 0.09$ & $\{\top, \perp\}: 0.03$ & $\{\mathrm{~T}, \perp\}: 0.03$ & $\{\top, \perp\}: 0.12$ \\
\hline$\{a, b\}: 0.1$ & \{\}$: 0.01$ & $\{\top\}: 0.03$ & $\{\top\}: 0.01$ & $\{\perp\}: 0.01$ & $\{\top, \perp\}: 0.04$ \\
\hline$\{a, b, c\}: 0.5$ & \{\}$: 0.05$ & $\{T\}: 0.15$ & $\{\top\}: 0.05$ & $\{\top\}: 0.05$ & $\{\top\}: 0.2$ \\
\hline
\end{tabular}

Fig. 5. Showing the tableau for the semantic unification of the membership values of the two mass assignments in Equations 5 and 21 including contradiction.

The unification of the membership functions including contradiction is:

$$
\begin{aligned}
\mu \operatorname{m}_{A \uparrow B}(\{\}) & =0.09 & & \mu \operatorname{m}_{A \uparrow B}(\{T\})=0.59 \\
\mu \operatorname{m}_{A \uparrow B}(\{F\}) & =0.01 & & \mu \operatorname{m}_{A \uparrow B}(\{T, F\})=0.31
\end{aligned}
$$

Giving an interim contradiction value in Equation 22

$$
\begin{aligned}
\iota \operatorname{m}_{A \uparrow B}(\emptyset) & =0.01 \\
\iota \operatorname{m}_{A \uparrow B}(\{T\}) & =0.11 \quad \iota \operatorname{m}_{A \uparrow B}(\{T, F\})=0.07
\end{aligned}
$$

The non-membership values for Equations 5 and 21

$$
\begin{aligned}
\text { Equation 5 } & \text { Equation 21 } \\
\nu \mathrm{m}_{A}(\{\})=0.6 & \nu \mathrm{m}_{B}(\{\})=0.6 \\
\nu \mathrm{m}_{A}(\{c\})=0.1 & \nu \mathrm{m}_{B}(\{c\})=0.1 \\
\nu \mathrm{m}_{A}(\{b, c\})=0.2 & \nu \mathrm{m}_{B}(\{b, c\})=0.2
\end{aligned}
$$

The possibility values derived from the non-membership values for Equations 5 and 21

$$
\begin{aligned}
\text { Equation 5 } & \text { Equation 21 } \\
\neg \nu \mathrm{m}_{A}(\{a, b, c\})=0.6 & \neg \nu \mathrm{m}_{B}(\{a, b, c\})=0.6 \\
\neg \nu \mathrm{m}_{A}(\{a, b\})=0.1 & \neg \nu \mathrm{m}_{B}(\{a, b\})=0.1 \\
\neg \nu \mathrm{m}_{A}(\{a\})=0.2 & \neg \nu \mathrm{m}_{B}(\{a\})=0.2
\end{aligned}
$$

The unification of the possibility values gives:

$$
\neg \nu \operatorname{m}_{\left.A\right|_{\neg \nu} B}(\{T\})=0.61 \quad \neg \nu \operatorname{m}_{\left.A\right|_{\neg \nu} B}(\{T, F\})=0.20
$$

The mass assignment of the non-membership values is:

$$
\nu \operatorname{m}_{\left.A\right|_{\nu} B}(\{F\})=0.61 \quad \nu \operatorname{m}_{\left.A\right|_{\nu} B}(\{\})=0.20
$$

The non-membership functions including contradictory evidence for Equations 5 and 21:

$$
\begin{aligned}
\text { Equation 5 } & \text { Equation 21 } \\
\nu \iota \mathrm{m}_{A}(\{\})=0.6 & \nu \iota \mathrm{m}_{B}(\{\})=0.6 \\
\nu \iota \mathrm{m}_{A}(\{a\})=0.1 & \nu \iota \mathrm{m}_{B}(\{a\})=0.1 \\
\nu \iota \mathrm{m}_{A}(\{c\})=0.1 & \nu \iota \mathrm{m}_{B}(\{c\})=0.1 \\
\nu \iota \mathrm{m}_{A}(\{b, c\})=0.2 & \nu \iota \mathrm{m}_{B}(\{b, c\})=0.2
\end{aligned}
$$

The possibility values derived from the non-membership values for Equations 5 and 21

$$
\begin{aligned}
\text { Equation 5 } & \text { Equation 21 } \\
\nu \iota \mathrm{m}_{A}(\{a, b, c\})=0.6 & \nu \iota \mathrm{m}_{B}(\{a, b, c\})=0.6 \\
\nu \iota \mathrm{m}_{A}(\{b, c\})=0.1 & \nu \iota \mathrm{m}_{B}(\{b, c\})=0.1 \\
\nu \iota \mathrm{m}_{A}(\{a, b\})=0.1 & \nu \iota \mathrm{m}_{B}(\{a, b\})=0.1 \\
\nu \iota \mathrm{m}_{A}(\{a\})=0.2 & \nu \iota \mathrm{m}_{B}(\{a\})=0.2
\end{aligned}
$$

The mass assignment resulting from the unification of the possibility values is:

$$
\begin{aligned}
\neg \nu \operatorname{m}_{\left.A\right|_{\neg \nu \iota} B} & (\{T\})=0.68 \quad \neg \nu \operatorname{m}_{\left.A\right|_{\neg \nu \iota} B}(\{F\})=0.04 \\
\neg \nu \underset{\left.A\right|_{\neg \nu \iota} B}{\mathrm{~m}}(\{T, F\}) & =0.28
\end{aligned}
$$

The mass assignment of the non-membership values is:

$$
\begin{gathered}
\nu \operatorname{m}_{\left.A\right|_{\nu \iota} B}(\{F\})=0.68 \quad \nu \operatorname{m}_{\left.A\right|_{\nu \iota} B}(\{T\})=0.04 \\
\nu \mathrm{m}_{\left.A\right|_{\nu \iota} B}(\{\})=0.28
\end{gathered}
$$

Giving a second interim contradiction value in Equation 23

$$
\begin{aligned}
& \iota \mathrm{m}_{\left.A\right|_{\iota} B}(\{F\})=0.07 \\
& \iota \mathrm{m}_{\left.A\right|_{\iota} B}(\{T\})=0.04 \quad \iota \mathrm{m}_{\left.A\right|_{\iota} B}(\{\})=0.08
\end{aligned}
$$

This is not the same as the contradictory values given by the membership analysis, however they are consistent with one another. We have the two assignments as shown in Equation 22 due to considerations of membership values and Equation 23 due to considerations of non-membership values.

The most general solution to this is the least upper bound of the two assignments which in this case is given in Equation 24:

$$
\begin{aligned}
\iota \mathrm{m}_{\left.A\right|_{\iota} B}(\{\}) & =0.01 & \iota \mathrm{m}_{\left.A\right|_{\iota} B}(\{F\})=0.0 \\
\iota \mathrm{m}_{\left.A\right|_{\iota} B}(\{T\}) & =0.04 \quad & \iota \mathrm{m}_{\left.A\right|_{\iota} B}(\{T, F\})=0.14
\end{aligned}
$$

The semantic unification of the mass assignments in Equations 5 and 21 results in the final triple mass assignment in Equation 25. 


$$
\begin{aligned}
\mathrm{m}_{A \mid B}(\{\}) & =\langle 0.08,0.20,0.01\rangle \\
\mathrm{m}_{A \mid B}(\{F\}) & =\langle 0.01,0.61,0.0\rangle \\
\mathrm{m}_{A \mid B}(\{T\}) & =\langle 0.48,0.0,0.04\rangle \\
\mathrm{m}_{A \mid B}(\{T, F\}) & =\langle 0.24,0.0,0.14\rangle
\end{aligned}
$$

\section{B. Semantic Separation}

Starting with the mass assignment from the unification in Equation 25 we evaluate how well this separates given the mass assignment in Equation 5. This inverts the process described in Section V-A.

The membership values for Equations 5 and 25

$$
\begin{aligned}
\text { Equation 5 } & \text { Equation 25 } \\
\mu \mathrm{m}_{A}(\{\})=0.1 & \mu \mathrm{m}_{A \mid B}(\{\})=0.08 \\
\mu \mathrm{m}_{A}(\{a\})=0.2 & \mu \mathrm{m}_{A \mid B}(\{F\})=0.01 \\
\mu \mathrm{m}_{A}(\{a, b\})=0.1 & \mu \mathrm{m}_{A \mid B}(\{T\})=0.48 \\
\mu \mathrm{m}_{A}(\{a, b, c\})=0.5 & \mu \mathrm{m}_{A \mid B}(\{T, F\})=0.24
\end{aligned}
$$

The mass assignment resulting from the separation of the membership functions is:

$$
\begin{aligned}
\mu \operatorname{m}_{A \uparrow A \mid B}(\emptyset) & =0.125 & & \mu \underset{A \uparrow A \mid B}{\operatorname{m}_{A}}(\{a\})=0.096 \\
\mu \operatorname{m}_{A \uparrow A \mid B}(\{a, b\}) & =0.048 & & \mu \operatorname{m}_{A \uparrow A \mid B}(\{c\})=0.001 \\
\mu \operatorname{m}_{A \uparrow A \mid B}(\{b, c\}) & =0.002 & & \mu \operatorname{m}_{A \uparrow A \mid B}(\{a, b, c\})=0.457
\end{aligned}
$$

The membership functions including contradictory evidence for Equations 5 and 25:

$$
\begin{aligned}
\text { Equation 5 } & \text { Equation 25 } \\
\mu \iota \mathrm{m}_{A}(\{\})=0.1 & \mu \iota \mathrm{m}_{A \mid B}(\{\})=0.156 \\
\mu \iota \mathrm{m}_{A}(\{a\})=0.3 & \mu \iota \mathrm{m}_{A \mid B}(\{F\})=0.01 \\
\mu \iota \mathrm{m}_{A}(\{a, b\})=0.1 & \mu \iota \mathrm{m}_{A \mid B}(\{T\})=0.52 \\
\mu \iota \mathrm{m}_{A}(\{a, b, c\})=0.5 & \mu \iota \mathrm{m}_{A \mid B}(\{T, F\})=0.38
\end{aligned}
$$

The mass assignment resulting from the separation of the membership functions including contradiction is:

$$
\begin{aligned}
\mu \operatorname{m}_{A \uparrow A \mid B}(\emptyset) & =0.125 & & \mu \operatorname{m}_{A \uparrow A \mid B}(\{a\})=0.156 \\
\mu \operatorname{m}_{A \uparrow A \mid B}(\{a, b\}) & =0.052 & & \mu \underset{A \uparrow A \mid B}{m_{A \uparrow}}(\{c\})=0.001 \\
\mu \operatorname{m}_{A \uparrow A \mid B}(\{b, c\}) & =0.003 & & \mu \underset{A \uparrow A \mid B}{m_{A}}(\{a, b, c\})=0.523
\end{aligned}
$$

Giving an interim contradiction value in Equation 26

$$
\begin{aligned}
\iota \mathrm{m}_{A \uparrow A \mid B}(\{a\}) & =0.06 \\
\iota \mathrm{m}_{A \uparrow A \mid B}(\{a, b\}) & =0.004 \quad \iota \operatorname{m}_{A \uparrow A \mid B}(\{b, c\})=0.001 \\
\iota \operatorname{m}_{A \uparrow A \mid B}(\{a, b, c\}) & =0.066
\end{aligned}
$$

The non-membership values for Equations 5 and 25

$$
\begin{aligned}
\text { Equation 5 } & \text { Equation 25 } \\
\nu \mathrm{m}_{A}(\{\})=0.6 & \nu \mathrm{m}_{A \mid B}(\{\})=0.2 \\
\nu \mathrm{m}_{A}(\{c\})=0.1 & \nu \mathrm{m}_{A \mid B}(\{F\})=0.61 \\
\nu \mathrm{m}_{A}(\{b, c\})=0.2 &
\end{aligned}
$$

The possibility values derived from the non-membership values for Equations 5 and 25

$$
\begin{aligned}
\text { Equation 5 } & \text { Equation 25 } \\
\neg \nu \mathrm{m}_{A}(\{a, b, c\})=0.6 & \neg \nu \mathrm{m}_{A \mid B}(\{\top, \perp\})=0.2 \\
\neg \nu \mathrm{m}_{A}(\{a, b\})=0.1 & \neg \nu \mathrm{m}_{A \mid B}(\{T\})=0.61 \\
\neg \nu \mathrm{m}_{A}(\{a\})=0.2 &
\end{aligned}
$$

The mass assignment resulting from the separation of the possibility values is:

$$
\begin{aligned}
& \neg \nu \operatorname{m}_{A \uparrow A \mid B}(\{a, b, c\})=0.366 \quad \neg \nu \operatorname{m}_{A \uparrow A \mid B}(\{a, b\})=0.061 \\
& \neg \nu \operatorname{m}_{A \uparrow A \mid B}(\{a\})=0.122 \quad \neg \nu \operatorname{m}_{A \uparrow A \mid B}(\{\})=0.18
\end{aligned}
$$

So the mass assignment of the non-membership values is:

$$
\begin{array}{rlrl}
\nu \operatorname{m}_{A \uparrow A \mid B}(\emptyset) & =0.366 & \nu \operatorname{m}_{A \uparrow A \mid B}(\{c\})=0.061 \\
\nu \operatorname{m}_{A \uparrow A \mid B}(\{b, c\}) & =0.122 \quad \nu \operatorname{m}_{A \uparrow A \mid B}(\{a, b, c\})=0.18
\end{array}
$$

The non-membership functions including contradictory evidence for Equations 5 and 25:

$$
\begin{aligned}
\text { Equation 5 } & \text { Equation 25 } \\
\nu \iota \mathrm{m}_{A}(\{\})=0.6 & \nu \iota \mathrm{m}_{A \mid B}(\emptyset)=0.21 \\
\nu \iota \mathrm{m}_{A}(\{a\})=0.1 & \nu \iota \mathrm{m}_{A \mid B}(\{F\})=0.61 \\
\nu \iota \mathrm{m}_{A}(\{c\})=0.1 & \nu \iota \mathrm{m}_{A \mid B}(\{T\})=0.04 \\
\nu \iota \mathrm{m}_{A}(\{b, c\})=0.2 & \nu \iota \mathrm{m}_{A \mid B}(\{T, F\})=0.14
\end{aligned}
$$

The possibility values derived from the non-membership 
values for Equations 5 and 25

$$
\begin{aligned}
\text { Equation 5 } & \text { Equation 25 } \\
\nu \iota \mathrm{m}_{A}(\{a, b, c\})=0.6 & \nu \iota \mathrm{m}_{A \mid B}(\{T, F\})=0.21 \\
\nu \iota \mathrm{m}_{A}(\{b, c\})=0.1 & \nu \iota \mathrm{m}_{A \mid B}(\{T\})=0.61 \\
\nu \iota \mathrm{m}_{A}(\{a, b\})=0.1 & \nu \iota \mathrm{m}_{A \mid B}(\{F\})=0.04 \\
\nu \iota \mathrm{m}_{A}(\{a\})=0.2 & \nu \iota \mathrm{m}_{A \mid B}(\emptyset)=0.14
\end{aligned}
$$

The result of the separation of the possibility values including contradiction is:

$$
\begin{aligned}
\neg \nu \iota \uparrow_{A \uparrow_{\nu \iota} A \mid B}(\{a, b, c\})=0.388 & \neg \nu \iota \operatorname{m}_{A \uparrow_{\nu \iota} A \mid B}(\{a\})=0.123 \\
\neg \nu \iota \mathrm{m}_{A \uparrow_{\nu \iota} A \mid B}(\{a, b\})=0.061 & \neg \nu \iota \mathrm{m}_{A \uparrow_{\nu \iota} A \mid B}(\{c\})=0.004 \\
\neg \nu \iota \mathrm{m}_{A \uparrow_{\nu \iota} A \mid B}(\{b, c\})=0.06 & \neg \nu \iota \operatorname{m}_{A \uparrow_{\nu \iota} A \mid B}(\{\})=0.364
\end{aligned}
$$

So the mass assignment of the non-membership values including contradiction is:

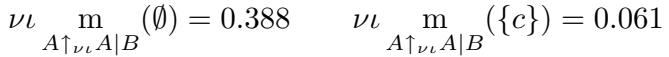

$$
\begin{aligned}
& \nu \iota \operatorname{m}_{A \uparrow_{\nu \iota} A \mid B}(\{b, c\})=0.123 \quad \nu \iota \operatorname{m}_{A \uparrow_{\nu \iota} A \mid B}(\{a\})=0.06 \\
& \nu \iota \operatorname{m}_{A \uparrow_{\nu \iota} A \mid B}(\{a, b\})=0.004 \quad \nu \iota{\underset{A \uparrow \nu}{\nu_{\nu \iota} A \mid B}}_{\mathrm{m}}(\{a, b, c\})=0.364
\end{aligned}
$$

Giving a second interim contradiction value in Equation 27

$$
\begin{aligned}
& \iota \operatorname{m}_{A \uparrow_{\iota} A \mid B}(\emptyset)=0.022 \\
& \iota \mathrm{m}_{A \uparrow_{\iota} A \mid B}(\{b, c\})=0.01 \quad \iota \operatorname{m}_{A \uparrow_{\iota} A \mid B}(\{a\})=0.06 \\
& \iota \mathrm{m}_{A \uparrow_{\iota} A \mid B}(\{a, b\})=0.004 \quad \iota_{A \uparrow_{\iota} A \mid B}(\{a, b, c\})=0.184
\end{aligned}
$$

As before we calculate the least upper bound of the two assignments given in Equation 28:

$$
\begin{array}{rlrl}
\iota & \mathrm{m}_{A \uparrow_{\iota} A \mid B}(\emptyset) & =0.014 \\
\iota \mathrm{m}_{A \uparrow_{\iota} A \mid B}(\{b, c\}) & =0.004 \quad \iota \operatorname{m}_{A \uparrow_{\iota} A \mid B}(\{a\})=0.069 \\
\iota \mathrm{m}_{A \uparrow_{\iota} A \mid B}(\{a, b\}) & =0.004 \quad \iota_{A \uparrow_{\iota} A \mid B}(\{a, b, c\})=0.054
\end{array}
$$

The semantic separation of the mass assignments in Equations 5 and 25 results in the final triple mass assignment in Equation 29.

$$
\begin{aligned}
\mathrm{m}_{A \uparrow A \mid B}(\{\}) & =\langle 0.125,0.366,0.014\rangle \\
\mathrm{m}_{A \uparrow A \mid B}(\{b, c\}) & =\langle 0.002,0.122,0.004\rangle \\
\operatorname{m}_{A \uparrow A \mid B}(\{a\}) & =\langle 0.096,0.0,0.069\rangle \\
\operatorname{m}_{A \uparrow A \mid B}(\{c\}) & =\langle 0.001,0.061,0.0\rangle \\
\operatorname{m}_{A \uparrow A \mid B}(\{a, b\}) & =\langle 0.048,0.0,0.004\rangle \\
\operatorname{m}_{A \uparrow A \mid B}(\{a, b, c\}) & =\langle 0.457,0.0,0.054\rangle
\end{aligned}
$$

VI. Conclusions

Non-membership values are usually computed using an s-norm if the main operator was a t-norm, and vice versa, however semantic unification and separation do not have a corresponding operator and so the possibility curve derived from the non-memberships was used, resulting in possibility values for the result which then gives the non-membership values. Semantic unification and separation have been introduced to Intuitionistic fuzzy Sets with a new method for computing the non-membership curves.

\section{References}

[1] K. Atanassov, "Intuitionistic fuzzy sets," Bulgarian Academy of Sciences, Tech. Rep. 1697/84, 1983.

[2] — , "Intuitionistic fuzzy sets," Fuzzy Sets and Systems, vol. 20, pp. 87-96, 1986.

[3] — Intuitionistic Fuzzy Sets: Theory and Applications. Heidelberg: Springer-Physica-Verlag, 1999.

[4] J. Baldwin, T. Martin, and B. Pilsworth, Fril - Fuzzy and Evidential Reasoning in Artificial Intelligence. Taunton UK: Research Studies Press Ltd, 1995.

[5] R. Patching, C. Hinde, and S. McCoy, "Inconsistency and semantic unification," Fuzzy Sets and Systems, vol. 157, pp. 2513-2539, 2006.

[6] C. Hinde, R. Patching, R. Stone, D. Xhemali, and S. McCoy, "Reasoning consistently about inconsistency," in Proceedings of 2007 IEEE International Conference on Fuzzy Systems, J. Garibaldi and P. Angelov, Eds., 2007, pp. 769-775.

[7] C. Hinde and R. Patching, Inconsistent Intuitionistic Fuzzy Sets. EXIT, 2007.

[8] C. Hinde, R. Patching, and S. McCoy, Inconsistent Intuitionistic Fuzzy Sets and Mass Assignment. EXIT, 2007.

[9] —-, "Managing contradictory evidence," in Proceedings of 2008 IEEE International Conference on Fuzzy Systems, 2008.

[10] J. Baldwin, "A theory of mass assignments for artificial intelligence," in Fuzzy Logic and Fuzzy Control, IJCAI'91 Workshops on fuzzy logic and fuzzy control. Sydney, Australia: Springer-Verlag, Jan 1991, pp. 2234.

[11] S. Cubillo and E. Castiñeira, "Contradiction in intuitionistic fuzzy sets," in Proceedings of the conference IPMU'2004. Perugia, Italy, 2004, pp. 2180-2186. 